Check for updates

Cite this: Chem. Sci., 2019, 10, 10503

¿ All publication charges for this article have been paid for by the Royal Society of Chemistry

Received 15th July 2019

Accepted 8th September 2019

DOI: $10.1039 /$ c9sc03501j

rsc.li/chemical-science

\section{Electronic coherence lifetimes of the Fenna- Matthews-Olson complex and light harvesting complex II $\dagger$}

\author{
Shawn Irgen-Gioro, (D) ${ }^{a}$ Karthik Gururangan, (D) a Rafael G. Saer, ${ }^{\text {b }}$ \\ Robert E. Blankenship (DD ${ }^{b}$ and Elad Harel ${ }^{\star a c}$
}

\begin{abstract}
The study of coherence between excitonic states in naturally occurring photosynthetic systems offers tantalizing prospects of uncovering mechanisms of efficient energy transport. However, experimental evidence of functionally relevant coherences in wild-type proteins has been tentative, leading to uncertainty in their importance at physiological conditions. Here, we extract the electronic coherence lifetime and frequency using a signal subtraction procedure in two model pigment-protein-complexes (PPCs), light harvesting complex II (LH2) and the Fenna-Matthews-Olson complex (FMO), and find that the coherence lifetimes occur at the same timescale (<100 fs) as energy transport between states at the energy level difference equal to the coherence energy. The pigment monomer bacteriochlorophyll a (BChla) shows no electronic coherences, supporting our methodology of removing long-lived vibrational coherences that have obfuscated previous assignments. This correlation of timescales and energy between coherences and energy transport reestablishes the time and energy scales that quantum processes may play a role in energy transport.
\end{abstract}

\section{Introduction}

The timescale in which a system maintains phase correlation, or coherence lifetime, dictates the observation of quantum interference at a macroscopic level. ${ }^{1}$ For electronic states, stochastic energy fluctuations cause coherences between the ground and the excited states to decay on the order of $10^{-14}$ seconds. In the late 2000 's, the assumption that coherences between two excited electronic states should be on a comparable timescale was challenged when a series of experiments observed coherences for hundreds of femtoseconds, with possible functional implications for energy transport in photosynthetic systems..$^{2-8}$ These initial experiments disputed the notions that the excited states' independent interaction with the thermal bath would rapidly destroy coherences between excitonic states (electronic coherences) and the conventional description of energy transfer using standard and modified Förster theory. ${ }^{9,10}$ However, more recent work has suggested that the spectroscopic signatures that began this debate,

${ }^{a}$ Department of Chemistry, Northwestern University, 2145 Sheridan Rd., Evanston IL 60208, USA

${ }^{b}$ Department of Biology, Washington University in St. Louis, One Brookings Dr St. Louis, MO 63130, USA

'Department of Chemistry, Michigan State University, East Lansing, Michigan 48824, USA. E-mail: harelela@msu.edu

$\dagger$ Electronic supplementary information (ESI) available: Sample growth and isolation, spectroscopic setup, simulated spectra used to verify subtraction procedure, additional figures. See DOI: 10.1039/c9sc03501j namely long-lived (hundreds of femtoseconds to picoseconds dephasing rate) coherences, can been attributed to originate from vibrations of the chromophores (vibrational coherences). ${ }^{11-15}$ In order to re-establish the timescale in which quantum effects may play a role in photosynthetic proteins, our study details a systematic method to extract electronic coherence lifetimes applied to two photosynthetic proteins, light harvesting complex 2 (LH2) and Fenna-Matthews-Olson (FMO) complex, using Multi-Dimensional Electronic Spectroscopy $(n \mathrm{DES})$.

The $n$ DES signal is generated with a series of three compressed broadband laser pulses, measured as a function of delay between the pulses, generating a 3D dataset. Two of these dimensions measure coherences between the ground and the excited state, which are popularly interpreted as the absorption frequency, $\omega_{\tau}$, and the emission frequency, $\omega_{t}$. The "population time", $T$, contains both population dynamics and the coherences between states within the excited or ground state manifold, which are the focus of this study and are detected as oscillations of the signal as a function of $T$. A recent theoretical study by Gelin et al. clearly partitions how electronic and vibrational degrees-of-freedom contribute to coherences at different timescales. ${ }^{16}$ At a $<100$ fs timescale, the time evolution is independent of nuclear degrees-of-freedom and is exclusively determined by electronic coupling. On a longer timescale, delocalized vibrations coupled electronic states form "vibronic" coherences, which eventually turn into purely vibrational coherences after a critical time $\tau_{D} \cong 2 \pi / \sigma$, where $\sigma$ is the 
dispersion of the static disorder. Understanding experimentally exactly when the signal transitions between different regimes of coherences gives microscopic details on the system-bath coupling. With a theoretical basis for what we expect to observe, our study aims to re-establish the timescale of electronic coherence in model photosynthetic proteins.

Previous studies have assigned large amplitude coherences that dephase at a $\sim 100$ fs timescale to be electronic coherences. ${ }^{15,17-19,20-22}$ However, the multitude of overlapping signals at early timepoints makes it difficult to extract values of the frequency and dephasing rate of the electronic coherence. The most common method currently to extract these parameters is to try to extrapolate the frequency based off estimating the period of oscillation, but trying to approximate the frequency of a damped oscillator this way is fraught with errors, especially in the case spectral congestion. The experimental measurement of electronic coherence lifetime and frequency is further complicated because the time domain signals are convolved with the instrument response correlation time. It is known that laser power and phase fluctuations follow a $1 / f$ distribution, leading to pulses at long time intervals to become increasingly uncorrelated. ${ }^{20}$ Thus, if a scan takes too long to acquire, coherence lifetimes will be artificially shortened. Previous measurements done by the corresponding author on LH2 showed coherences decaying with a $\sim 100$ fs lifetime, ${ }^{3}$ but our current measurements see the same coherences out to many picoseconds as a result of the fast measurement, in which the entire scan is completed before significant loss of pulse correlation (typically, less than $2 \mathrm{~s}$ ). Our study combines the rapid acquisition experimental methodology, which provides high SNR data, with a systematic procedure to extract electronic coherence to overcome these challenges.

\section{Results}

$n$ DES spectra are taken for BChla, LH2, and FMO, and in the case of LH2 and FMO a bimodal distribution of coherence lifetimes is observed. The long-lived coherences show agreement with a recent control experiment measured in our lab of vibrational coherences in BChla and gives us confident assignment of long-lived coherences as primarily vibrational in origin. ${ }^{13}$ The contributions from population dynamics and vibrational coherences are removed with global analysis. ${ }^{21}$ Briefly, the 3D spectrum is written as a matrix multiplication of a basis set of real or complex exponentials and its amplitude for each point in the $2 \mathrm{D}$ spectra. Variable projection is used to find the basis set that best describes the full spectra, which essentially decomposes the spectrum into the contributions from each of the basis functions, allowing for reconstruction of spectra from a subset of components. This procedure is first run with a basis set of real exponentials, and the reconstructed spectra is subtracted to remove the contribution from population dynamics. The residual is run through global analysis again using a basis set of complex exponentials. In the second global analysis run, the early time points are excluded, and only later time points are considered. Thus, the reconstructed spectrum contains contributions only from the long-lifetime coherences. The resulting spectra is then subtracted from the population subtracted spectra to reveal the rapidly dephasing coherences in the typically congested first few hundred femtoseconds. This systematic method of subtracting vibrations is insensitive to the choice of cut-off time as long as one chooses a time longer than the electronic coherence lifetime because they have negligible contribution to the signal at long time points. We recently used a similar method to remove contributions to the signal from solute-only third order response and is described in more detail in this context in the methods section. ${ }^{13}$ Fig. 1 diagrammatically shows how the vibration subtraction procedure is performed in LH2. The real rephasing

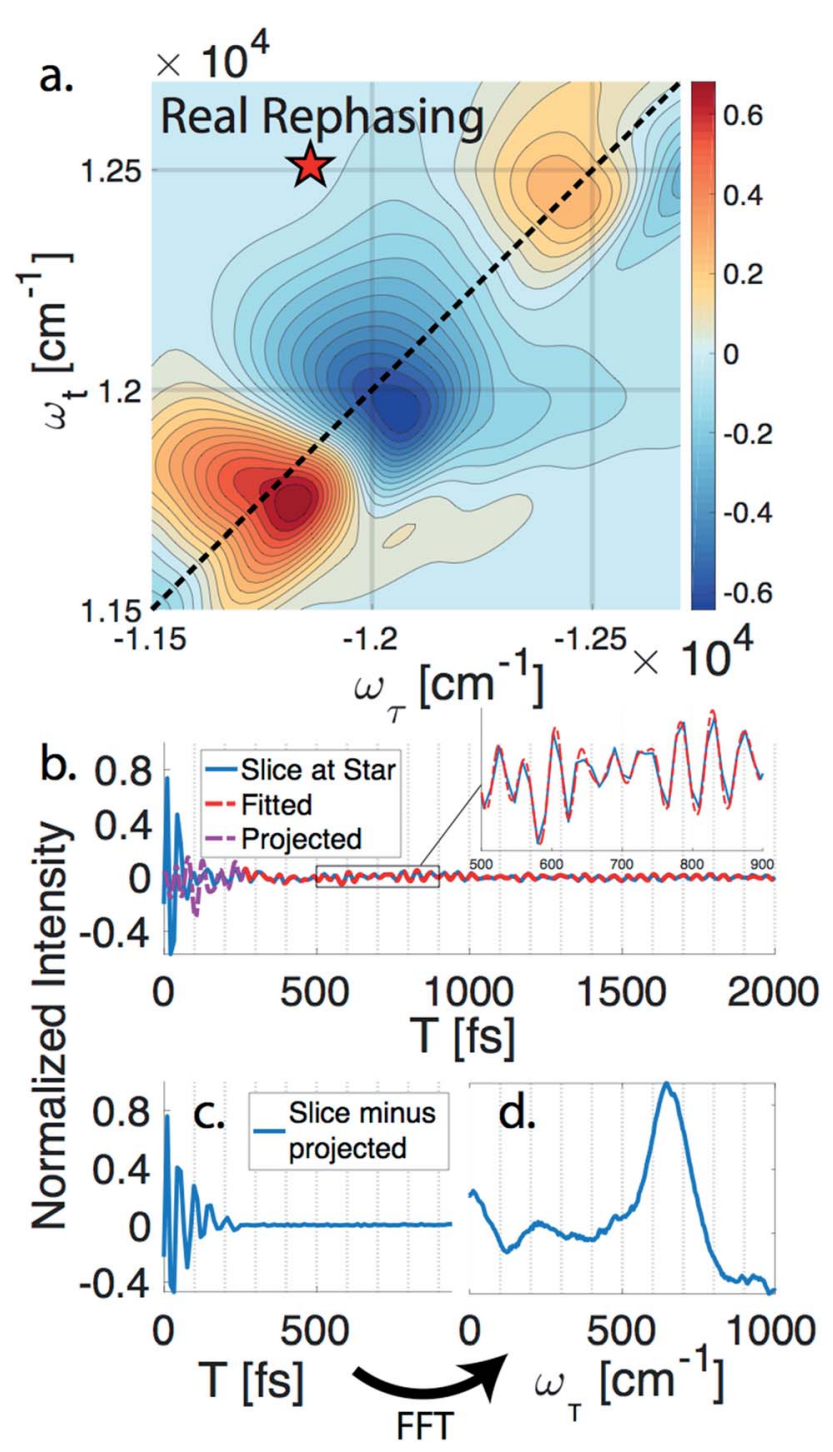

Fig. 1 Methodology of extracting electronic coherence from the light harvesting complex II. (a) A slice of the real rephasing $\mathrm{LH} 2$ 3DES spectrum is shown at $T=43 \mathrm{fs}$. (b) An example point from (a) is chosen at the star with population dynamics subtracted using global analysis. The residual (blue) is fit for later time points (red) and projected back to the early time points (purple). (c) The short lifetime coherences are revealed once the projected coherences are subtracted. (d) A Fourier transform is performed on (c) to reveal the line width and coherence frequency. 
spectra is shown in (a) at $T=43$ fs. Once the population dynamics are removed, each point in the $2 \mathrm{D}$ spectrum contains oscillations as a function of $T$, which are the electronic and vibrational coherences (b). The long-lifetime coherences are fit at later time points and projected back to $T=0$ with their respective amplitudes found through global analysis. Subtracting these contributions from long-lived coherences reports on the electronic coherences (c). These short-lived coherences can then be Fourier transformed to reveal the line width and coherence frequency $(\mathrm{d})$.

The vibrational subtraction analysis is first performed on $\mathrm{BChl} a$, which is the monomer pigment found in both $\mathrm{LH} 2$ and FMO. Coherences of BChla must be vibrational in origin since there is only one accessible excited electronic state within our pulse bandwidth. The sum of the Fourier transformed residual, like the one seen in Fig. 1(d), for the entire 2D spectrum can be

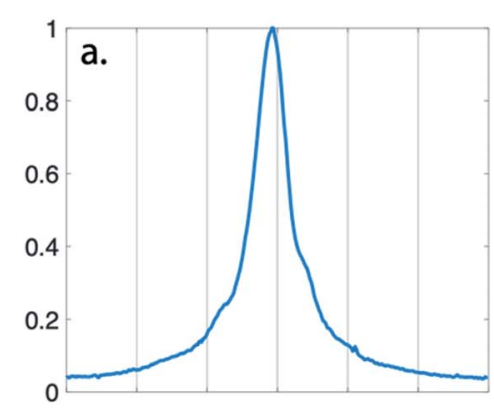

\section{Bacterio- chlorophyll a}
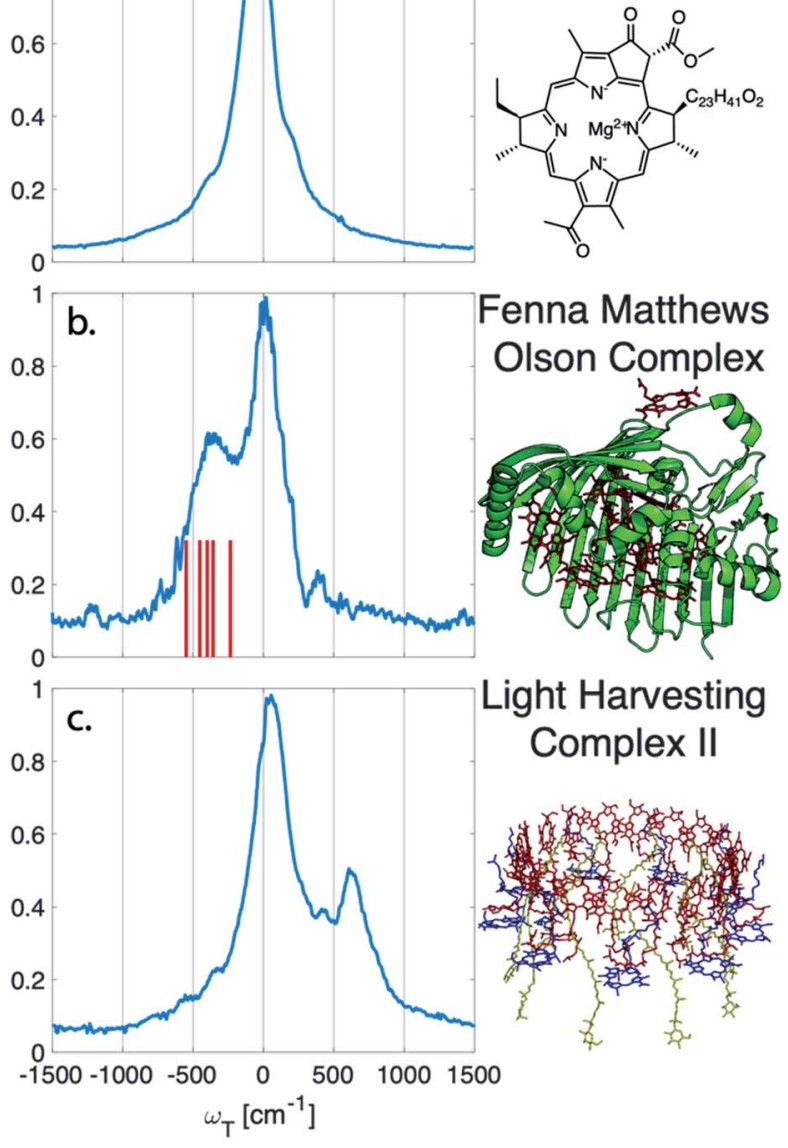

Fig. 2 Sum of the electronic coherences over the entire 2D spectrum. (a) The BChla spectra contains only vibrational coherences. With the long-lived coherences subtracted, no additional peaks are observed. Both (b) FMO and (c) LH2 have multiple electronic states within the bandwidth of the pulse and display fast decaying coherences. For FMO, excitonic energy level differences are drawn as a stick spectrum for differences between 330-550 $\mathrm{cm}^{-1}$. The closest excitonic energy level difference to the main peak is between excitons 7 and 2 at $400 \mathrm{~cm}^{-1}$, with the energy levels taken from ref. 22 . seen in Fig. 2. This analysis on BChla shows no remaining electronic coherence peaks, only a background that comes primarily from imperfect subtraction of real exponentials, nonexponential signals, and remaining $1 / f$ noise.

Unlike BChla where no electronic coherences are possible, LH2 and FMO may show both electronic and vibrational coherences owing to the high-density of excitonic states within the pulse bandwidth. The same subtraction procedure applied on LH2 and FMO, as seen in Fig. 2(b and c), show broad peaks. For FMO, the main peak is centred around $\sim 350 \mathrm{~cm}^{-1}$. FMO is a system of $8 \mathrm{BChl}$, with one weakly surface bound BChl likely to become disassociated during the process of isolation and purification. The seven remaining excitonic states have energies ranging from 12120 to $12670 \mathrm{~cm}^{-1}$ and the energy level difference of some of the excitons are displayed as sticks in Fig. 2(b). ${ }^{22}$ The closest excitonic energy level difference to the observed peak is between excitons 7 and 2 using the numbering of BChls chosen by Fenna and Matthews. ${ }^{23}$ It is thought that excitons are delocalized across $1 / 2,3 / 4$, and 5/6/7, and even though excitons 7 and 2 are neighbouring chromophores, they are predicted to have relatively weak excitonic coupling. ${ }^{24}$ Previous reports of rapidly dephasing coherences in FMO found that the lowest oscillation frequency was well above the distribution of excitonic energy level differences $\left(\sim 600 \mathrm{~cm}^{-1}\right)$, not matching any exciton energy differences. ${ }^{15}$ In LH2, the electronic coherence extracted is centred at $\sim 630 \mathrm{~cm}^{-1}$. However, since the excitonic energies are ambiguous, no proposed energy level differences are drawn. The main complicating factor is the high density of excitonic states with similar energies that can be modulated by specific instances of disorder. ${ }^{25}$ From the absorption spectrum, the energy level difference of the two $\mathrm{BChl} a$ rings, B800 and B850, is $735 \mathrm{~cm}^{-1}$. Previous work on a mutant of $\mathrm{LH} 2$ with the $\mathrm{B} 800$ band removed observed an electronic coherence at $687 \mathrm{~cm}^{-1}$, which was assigned to be between $\mathrm{B} 850$ and a higher lying excitonic state B850*.17 Although there is uncertainty to which states the observed coherences are between, it is clear that both FMO and LH2 contain rapidly dephasing coherences that do not exist in BChla.

Global analysis with a complex exponential basis retrieves the lifetime and energy of the electronic coherence peaks. For LH2 the main peak is centred at $680 \mathrm{~cm}^{-1}$ with a lifetime of 81 fs, and for FMO it is at $390 \mathrm{~cm}^{-1}$ with a lifetime of $57 \mathrm{fs}$. However, we find that global analysis sometimes has difficulty in retrieving lifetimes of broadened peaks and is sensitive to the number of exponentials used to fit a given peak. Thus, the lifetimes extracted with global analysis are supplemented with the 1/e decay rates from the time domain of individual points in the $2 \mathrm{D}$ spectra. Estimating the electronic decoherence rates from the time traces, like the one seen in Fig. 1(c), gives $\sim 80$ and $\sim 60$ fs dephasing rates for the largest peaks of LH2 and FMO, respectively. These dephasing rates can be compared to the expected dephasing rates, which are revealed using the antidiagonal slice of the $2 \mathrm{D}$ spectrum. The antidiagonal slice reveals the homogenous broadening of a state, which is related to the dephasing time between the ground and excited state by $\tau=$ $[\pi c \Delta]^{-1}$, where $\Delta$ is the FWHM of a fitted Lorentzian. This holds 
true even in the presence of strong vibronic coupling. ${ }^{26}$ While FMO's expected dephasing rate matches the coherence lifetime, the estimated lifetimes of the B800 and B850 states in LH2 gives 56 and 42 fs respectively (slices and fits shown in ESI $\dagger$ ). The measured electronic coherence lifetime of $\sim 80$ fs is unexpectedly longer than the dephasing rate of the individual states. Gelin et al. offers another method to predict the coherence lifetime using the expression $\tau^{-2}=\sum_{k=1}^{N} J_{1 k}^{2}$, where $N$ is the number of chromophores and $J$ is the inter-site electronic coupling strength. ${ }^{16}$ Comparison to their predicted value of 53 fs of the electronic coherence lifetime of FMO, we find good agreement. However for LH2, which has more chromophores and higher coupling strength (at least in the B850 band), we should expect the coherence lifetime to be even shorter. ${ }^{32}$ This trend holds true for the rates extracted from the anti-diagonal slices, but not for the electronic coherence measured in the time domain. Using both metrics, we find that the measured electronic coherence lifetime is longer than predicted. The electronic coherence frequency and lifetimes obtained from our methodology are summarized in Table 1.

Since our experiment establishes that the electronic coherences are happening at a $<100 \mathrm{fs}$ timescale, for coherences to be functional and not simply a phenomenon universal to all molecules there has to be biologically relevant processes happening on the same timescale. For FMO, the kinetic rates between all sites has been well characterized and shows that this timescale is irrelevant for exciton transport. ${ }^{22}$ However, in LH2, <100 fs timescale processes have been implicated in both energy transfer between the rings through intermediate states and population transfer to states with mixed excitonic and charge transfer character process. ${ }^{27-29}$ Thus, we will take a closer look at the population dynamics extracted during the first iteration of global analysis. This viewed the signal as a sum of contributions from real exponentials, $\mathrm{e}^{-t / \tau}$, described with a single parameter, which is the decay lifetime $\tau$. The associated amplitude for a given exponential decay at each point on the 2D spectra is called the Decay Associated Spectra (DAS) beatmap and shows all species that have dynamic components over $T$ with a given rate. The difficulties of interpreting DAS in general are explored in the ESI, $\dagger$ but interpretation of the BChl $a$ spectra is simplified because it only contains one electronic excited state with simpler dynamical processes compared to FMO or LH2. Furthermore, the BChla 2D spectrum contains no contributions from ESA, so positive signal on the DAS beatmap indicates a decay of signal while negative areas indicate signal growth. In Fig. 3, it can be seen that the DAS for BChla and LH2
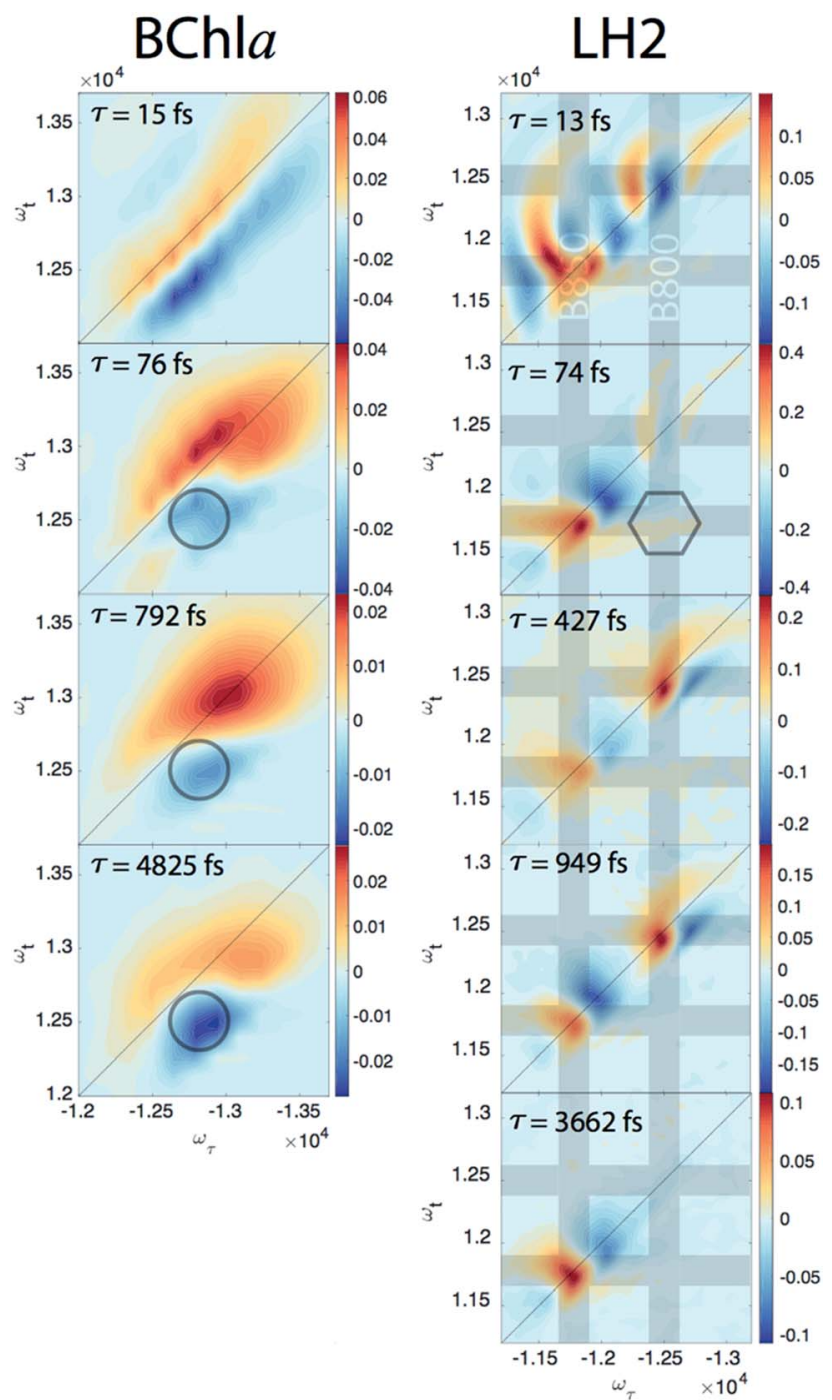

Fig. 3 DAS beatmaps of BChla (left) and LH2 (right). The BChla assignments are aided by only having one electronic state within the bandwidth of the laser and simpler dynamic processes. The BChla 13 fs component indicates a global shift energy and is attributed to a Stokes shift convolved with instrument response. The 76 fs component appears to be an internal relaxation process with signal growth at the low energy side of the spectra (highlighted in the circle). The $74 \mathrm{fs} \mathrm{LH2}$ component most likely has similar internal relaxation processes, but also appears to have amplitude at the downhill cross-peak $\left[\omega_{\tau}, \omega_{\tau}\right]=$ $[-12400,11700] \mathrm{cm}^{-1}$, located in the hexagon, at roughly the electronic coherence energy. Grey bands are located on the energies of the $\mathrm{B} 800$ and $\mathrm{B} 850$ states.

Table 1 Comparison of electronic coherence lifetime and frequency extracted through global and Fourier analysis

\begin{tabular}{llllll}
\hline Protein & Global Lifetime & Global Frequency & Fourier Lifetime & Fourier Frequency & Expected Dephasing \\
\hline FMO $^{a}$ & $57 \mathrm{fs}$ & $390 \mathrm{~cm}^{-1}$ & $60 \mathrm{fs}$ & $350 \mathrm{~cm}^{-1}$ & $60 \mathrm{fs}$ \\
LH2 $^{b 17}$ & $81 \mathrm{fs}$ & $680 \mathrm{~cm}^{-1}$ & $80 \mathrm{fs}$ & $630 \mathrm{~cm}^{-1}$ & $42-56 \mathrm{fs}$
\end{tabular}

${ }^{a}$ Previous work saw coherences on $\sim 100$ fs timescale but did not extract rates or energies. ${ }^{b}$ Previous work on mutated LH2 with the B800 band removed found electronic coherences at $680 \mathrm{~cm}^{-1}$ with a lifetime of $137 \mathrm{fs}$. 
both possess similar fast ( $<100 \mathrm{fs}$ ) components. In the BChla 15 fs component, the signal grows in on the Stokes shifted side of the diagonal while decaying on the anti-Stokes side, indicating a global shift of energies. However, since this component is on the timescale of our pulse duration, we assign this to instrument response. The other components of the BChla indicate growth of signal pumping at higher energies and probing at lower energies, $\left[\omega_{\tau}, \omega_{\tau}\right]=[-12800,12500] \mathrm{cm}^{-1}$, indicated on Fig. 3 with circles. This growth of signal at the lowest energy state suggests these components are internal relaxation processes. Utilizing the BChl $a$ assignments to inform the DAS beatmaps in $\mathrm{LH} 2$, the 15 fs component is similarly assigned to be instrument response. However, for the $\mathrm{LH} 274$ fs component, non-internal relaxation processes distinct from the BChla 76 fs DAS beatmap appear. Amplitude at the downhill cross-peak at $\left[\omega_{\tau}, \omega_{t}\right]=[-12400,11700] \mathrm{cm}^{-1}$, highlighted on Fig. 3 in the hexagon, indicating a pump and probe energy level difference of $\sim 700 \mathrm{~cm}^{-1}$, shows energy transfer at the same timescale and energy as the electronic coherence. Dynamic processes that may be occurring at a $<\sim 100$ fs timescale are discussed later, but it is possible that the same state in coherence are experiencing energy transfer at this ultrafast timescale. The slower rates of LH2 are attributed to decay of the ground state bleach of the B800 and B850 rings and population transfer between the two rings. Specifically, the $\sim 3.6$ ps decay shows up only on the B850 band of LH2 and the broad bi-excitonic ESA feature. The similar lifetimes, also seen in pump-probe experiments, have been used to justify that the ESA originates from the excited B850 ring. ${ }^{30}$ The other two slower 472 and 949 fs components are difficult to assign due to the similarity of features. It is possible that they are the same component, which would agree with previous reports have indicated that the $\mathrm{B} 800$ and $\mathrm{B} 850$ transfer rate occurs with $\tau=700$ fs. $^{31}$

\section{Discussion}

Identifying the lifetime and energy of electronic coherences limits the role of electronic coherences to the $<100$ fs timescale and in turn what biological functions may be implicated. Although it is possible that electronic coherence coincidentally occurs at the same timescale as dynamic processes between states of the same energy level difference, the large body of work on LH2 provides clues to which states may be involved in the electronic coherence. One likely possibility is that the coherence observed here is between the B850 and B850* state, which would be the same coherence previously observed in the mutated species by the Engel group. ${ }^{17}$ B850* refers to the higher lying excited states of the B850 ring's excitonic progression. Although the typically named $k= \pm 1$ transition contains most of the total oscillator strength, symmetry breaking allows for other transitions that overlap the B800 band. This model predicts that energy transport within the B850 band occurs on a $<100$ fs timescale. ${ }^{29}$ Atomistic modelling of LH2 has shown that in the unmutated species, the B850* state mixes with the B800 state and that excitation becomes delocalized across both rings. This mixed state is thought to be an intermediate between the B800 and B850 ring, mediating stepwise downhill transport. ${ }^{28}$ Thus, coherences within the excitonic manifold of B850 offer explanations to both the $<100$ fs electronic coherence and energy transfer. However, another mutant study where the B850 band is shifted to $810 \mathrm{~nm}$ revealed a dark state with charge transfer character around $850 \mathrm{~nm},{ }^{27}$ but with no electronic coherences reported. This study revealed that excitations in the B800 band are transferred to the CT state in a $<100$ timescale. In the unmutated species, this CT state is theorized to mix with the B850 ring further adding energy levels to an already congested energy landscape. Due to the multitude of possible states involved, we cannot confidently assign the states in coherence purely based off the energy of the electronic coherence observed.

\section{Methods}

\section{Global analysis}

The 3D spectrum, $Y$, is a $N_{\tau} \times N_{T} \times N_{t}$ matrix. This can be rewritten as a matrix multiplication problem, $Y=G T$, where $G$ is a $N_{\tau} \times N_{t} \times m$ matrix and $T$ is a $m \times N_{T}$ matrix, and $m$ is the number of basis sets. $T$ is what is referred to as the basis set, as each $1 \times N_{T}$ row of the matrix is either a real or complex exponential depending on if populations or coherences are fit. $G$ is the associated amplitude of each basis. In the case of real exponentials, the $N_{\tau} \times N_{t} \times 1$ matrix associated with a given exponential is the DAS beatmap. Since $G$ can be solved for by multiplying the original data by a basis set, $G=Y T^{-1}$, the entire optimization can be boiled down to finding the basis set that best describes the data. Using variable projection, this minimizes the number of parameters needed to be solved. To fit later times, only data past a certain time point is considered so that the basis set now is described by a $m \times\left(N_{T}-N_{\text {exclude }}\right)$ matrix, where $N_{\text {exclude }}$ is the number of early time points no longer considered. To extrapolate, the $G$ from the late times is multiplied by a new basis set that now includes the early time points, keeping the same coefficients for each complex exponential basis. Our methodology of fitting later time points and projecting the fit back to the early time points is tested out on simulated spectra in the ESI. $\dagger$

\section{Sample isolation}

LH2 and BChla are isolated from Rhodobacter sphaeroides 2.4.1. FMO is extracted from Chlorobaculum tepidum. Details can be found in the ESI. $\dagger$

\section{Conclusions}

Observation of excitonic coherences in photosynthetic complexes on the timescale of biologically relevant dynamics constrains the time and energy scales that electronic coherences may play a role. Electronic decoherence lifetimes were extracted from FMO and LH2, while similar analysis on their pigment monomer, BChla revealed no early time coherences. While the majority of exciton transport in these systems happens on a picosecond timescale and is dominated by spatially directed dipole coupling, our study suggests that there may still be a niche function for electronic coherence at a $<100$ fs timescale. 


\section{Conflicts of interest}

There are no conflicts to declare.

\section{Acknowledgements}

We thank the recombinant protein production core, specifically Dr Sergii Pshenychnyi, at Northwestern University for their help, advice, and equipment lending. Also, we thank Prof. Pamela Parkes-Loach and Prof. Paul Loach in their guidance in growing bacteria. This work was supported by the Air Force Office of Scientific Research (FA9550-14-1-0005), and the Packard Foundation (2013-39272) in part.

\section{Notes and references}

$1 \mathrm{H}$. D. Zeh, On the interpretation of measurement in quantum theory, Found. Phys., 1970, 1(1), 69-76.

2 G. S. Engel, T. R. Calhoun, E. L. Read, T.-K. Ahn, T. Mančal, Y.-C. Cheng, R. E. Blankenship and G. R. Fleming, Evidence for wavelike energy transfer through quantum coherence in photosynthetic systems, Nature, 2007, 446, 782.

3 E. Harel and G. S. Engel, Quantum coherence spectroscopy reveals complex dynamics in bacterial light-harvesting complex 2 (LH2), Proc. Natl. Acad. Sci. U. S. A., 2012, 109(3), 706.

4 E. Collini, C. Y. Wong, K. E. Wilk, P. M. G. Curmi, P. Brumer and G. D. Scholes, Coherently wired light-harvesting in photosynthetic marine algae at ambient temperature, Nature, 2010, 463, 644.

5 M. B. Plenio and S. F. Huelga, Dephasing-assisted transport: quantum networks and biomolecules, New J. Phys., 2008, 10(11), 113019.

6 M. Mohseni, P. Rebentrost, S. Lloyd and A. Aspuru-Guzik, Environment-assisted quantum walks in photosynthetic energy transfer, J. Chem. Phys., 2008, 129(17), 174106.

7 A. Ishizaki and G. R. Fleming, Theoretical examination of quantum coherence in a photosynthetic system at physiological temperature, Proc. Natl. Acad. Sci. U. S. A., 2009, 106(41), 17255.

8 S. Lloyd, Quantum coherence in biological systems, J. Phys.: Conf. Ser., 2011, 302(1), 012037.

9 H. M. Wu, S. Savikhin, N. R. S. Reddy, R. Jankowiak, R. J. Cogdell, W. S. Struve and G. J. Small, Femtosecond and Hole-Burning Studies of B800's Excitation Energy Relaxation Dynamics in the LH2 Antenna Complex of Rhodopseudomonas acidophila (Strain 10050), J. Phys. Chem., 1996, 100(29), 12022-12033.

10 G. D. Scholes and G. R. Fleming, On the Mechanism of Light Harvesting in Photosynthetic Purple Bacteria: B800 to B850 Energy Transfer, J. Phys. Chem. B, 2000, 104(8), 1854-1868.

11 N. Christensson, H. F. Kauffmann, T. Pullerits and T. Mančal, Origin of Long-Lived Coherences in LightHarvesting Complexes, J. Phys. Chem. B, 2012, 116(25), 7449-7454.

12 M. B. Plenio, J. Almeida and S. F. Huelga, Origin of long-lived oscillations in 2D-spectra of a quantum vibronic model:
Electronic versus vibrational coherence, J. Chem. Phys, 2013, 139(23), 235102.

13 S. Irgen-Gioro, A. P. Spencer, W. O. Hutson and E. Harel, Coherences of Bacteriochlorophyll a Uncovered Using 3DElectronic Spectroscopy, J. Phys. Chem. Lett., 2018, 60776081.

14 M. Maiuri, E. E. Ostroumov, R. G. Saer, R. E. Blankenship and G. D. Scholes, Coherent wavepackets in the FennaMatthews-Olson complex are robust to excitonic-structure perturbations caused by mutagenesis, Nat. Chem., 2018, 10, 177.

15 H.-G. Duan, V. I. Prokhorenko, R. J. Cogdell, K. Ashraf, A. L. Stevens, M. Thorwart and R. J. D. Miller, Nature does not rely on long-lived electronic quantum coherence for photosynthetic energy transfer, Proc. Natl. Acad. Sci. U. S. A., 2017, 114(32), 8493.

16 M. F. Gelin, R. Borrelli and W. Domcke, Origin of Unexpectedly Simple Oscillatory Responses in the ExcitedState Dynamics of Disordered Molecular Aggregates, $J$. Phys. Chem. Lett., 2019, 10(11), 2806-2810.

17 V. P. Singh, M. Westberg, C. Wang, P. D. Dahlberg, T. Gellen, A. T. Gardiner, R. J. Cogdell and G. S. Engel, Towards quantification of vibronic coupling in photosynthetic antenna complexes, J. Chem. Phys., 2015, 142(21), 212446.

18 H.-G. Duan, A. L. Stevens, P. Nalbach, M. Thorwart, V. I. Prokhorenko and R. J. D. Miller, Two-Dimensional Electronic Spectroscopy of Light-Harvesting Complex II at Ambient Temperature: A Joint Experimental and Theoretical Study, J. Phys. Chem. B, 2015, 119(36), 1201712027.

19 E. Thyrhaug, R. Tempelaar, M. J. P. Alcocer, K. Žídek, D. Bína, J. Knoester, T. L. C. Jansen and D. Zigmantas, Identification and characterization of diverse coherences in the Fenna-Matthews-Olson complex, Nat. Chem., 2018, 10(7), 780-786.

20 Y. Feng, I. Vinogradov and N.-H. Ge, General noise suppression scheme with reference detection in heterodyne nonlinear spectroscopy, Opt. Express, 2017, 25(21), 26262-26279.

21 A. Volpato, L. Bolzonello, E. Meneghin and E. Collini, Global analysis of coherence and population dynamics in $2 \mathrm{D}$ electronic spectroscopy, Opt. Express, 2016, 24(21), 2477324785.

22 E. Thyrhaug, K. Žídek, J. Dostál, D. Bína and D. Zigmantas, Exciton Structure and Energy Transfer in the FennaMatthews-Olson Complex, J. Phys. Chem. Lett., 2016, 7(9), 1653-1660.

23 R. E. Fenna and B. W. Matthews, Chlorophyll arrangement in a bacteriochlorophyll protein from Chlorobium limicola, Nature, 1975, 258, 573.

24 J. Adolphs and T. Renger, How Proteins Trigger Excitation Energy Transfer in the FMO Complex of Green Sulfur Bacteria, Biophys. J., 2006, 91(8), 2778-2797.

25 A. M. van Oijen, M. Ketelaars, J. Köhler, T. J. Aartsma and J. Schmidt, Unraveling the Electronic Structure of Individual Photosynthetic Pigment-Protein Complexes, Science, 1999, 285(5426), 400. 
26 A. Halpin, P. J. M. Johnson, R. Tempelaar, R. S. Murphy, J. Knoester, T. L. C. Jansen and R. J. D. Miller, Twodimensional spectroscopy of a molecular dimer unveils the effects of vibronic coupling on exciton coherences, Nat. Chem., 2014, 6, 196.

27 M. Ferretti, R. Hendrikx, E. Romero, J. Southall, R. J. Cogdell, V. I. Novoderezhkin, G. D. Scholes and R. van Grondelle, Dark States in the Light-Harvesting complex 2 Revealed by Two-dimensional Electronic Spectroscopy, Sci. Rep., 2016, 6, 20834.

28 C. P. van der Vegte, J. D. Prajapati, U. Kleinekathöfer, J. Knoester and T. L. C. Jansen, Atomistic Modeling of Two-Dimensional Electronic Spectra and Excited-State Dynamics for a Light Harvesting 2 Complex, J. Phys. Chem. B, 2015, 119(4), 1302-1313.

29 J. Strümpfer and K. Schulten, Light harvesting complex II B850 excitation dynamics, J. Chem. Phys., 2009, 131(22), 225101.

30 L. Guo, Y. Liu, Y. Yang, J. Mi, C. Xu, C. Xu and S. Qian, The observation of excited-state dynamical evolution in light- harvesting complex LH2 from Rhodobacter sphaeroides 601, FEBS Lett., 2002, 511(1), 69-72.

31 G. R. Fleming and R. v. Grondelle, Femtosecond spectroscopy of photosynthetic light-harvesting systems, Curr. Opin. Struct. Biol., 1997, 7(5), 738-748.

32 S. Tretiak, C. Middleton, V. Chernyak and S. Mukamel, Exciton Hamiltonian for the Bacteriochlorophyll System in the LH2 Antenna Complex of Purple Bacteria, J. Phys. Chem. B, 2000, 104(18), 4519-4528.

33 E. Romero, et al., Quantum coherence in photosynthesis for efficient solar-energy conversion, Nat. Phys., 2014, 10, 676682.

34 F. D. Fuller, et al., Vibronic coherence in oxygenic photosynthesis, Nat. Chem., 2014, 6, 706-711.

35 F. Ma, E. Romero, M. R. Jones, V. I. Novoderezhkin and R. van Grondelle, Both electronic and vibrational coherences are involved in primary electron transfer in bacterial reaction center, Nat. Commun., 2019, 10(1), 933. 